\title{
(6) OPEN ACCESS \\ Advancing clinical development pathways for new CFTR modulators in cystic fibrosis
}

\author{
Nicole Mayer-Hamblett, ${ }^{1,2}$ Michael Boyle, ${ }^{3,4}$ Donald VanDevanter ${ }^{5}$
}

${ }^{1}$ Department of Pediatrics and Biostatistics, University of Washington, Seattle,

Washington, USA

${ }^{2}$ Seattle Children's Hospital,

Seattle, Washington, USA

${ }^{3}$ Cystic Fibrosis Foundation, Bethesda, Maryland, USA

${ }^{4}$ John Hopkins School of

Medicine, Baltimore, Maryland, USA

${ }^{5}$ Case Western Reserve University School of Medicine, Cleveland, Ohio, USA

\section{Correspondence to} Dr Nicole Mayer-Hamblett, Department of Pediatrics, University of Washington, 4800 Sand Point Way N.E., Box 5371, Seattle WA 981050371, USA

nicole.hamblett@

seattlechildrens.org

Received 24 November 2015 Revised 4 January 2016 Accepted 20 January 2016 Published Online First 22 February 2016

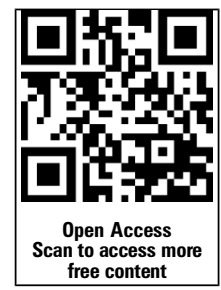

CrossMark

To cite: Mayer-Hamblett $\mathrm{N}$, Boyle $M$, VanDevanter D. Thorax 2016;71:454-461.

\section{ABSTRACT}

Cystic fibrosis (CF) is a life-shortening genetic disease affecting approximately 70000 individuals worldwide. Until recently, drug development efforts have emphasised therapies treating downstream signs and symptoms resulting from the underlying CF biological defect: reduced function of the $\mathrm{CF}$ transmembrane conductance regulator (CFTR) protein. The current CF drug development landscape has expanded to include therapies that enhance CFTR function by either restoring wild-type CFTR protein expression or increasing (modulating) the function of mutant CFTR proteins in cells. To date, two systemic small-molecule CFTR modulators have been evaluated in pivotal clinical trials in individuals with CF and specific mutant CFTR genotypes that have led to regulatory review and/or approval. Advances in the discovery of CFTR modulators as a promising new class of therapies have been impressive, yet work remains to develop highly effective, disease-modifying modulators for individuals of all CF genotypes. The objectives of this review are to outline the challenges and opportunities in drug development created by systemic genotype-specific CFTR modulators, highlight the advantages of sweat chloride as an established biomarker of CFTR activity to streamline early-phase development and summarise options for later phase clinical trial designs that respond to the adoption of approved genotype-specific modulators into standard of care. An optimal development framework will be needed to move the most promising therapies efficiently through the drug development pipeline and ultimately deliver efficacious and safe therapies to all individuals with CF.

\section{INTRODUCTION}

Cystic fibrosis (CF) is the most common lifeshortening genetic disease among Caucasians, yet affects only approximately 70000 individuals worldwide. ${ }^{1}$ The complexities of developing new therapies for rare diseases are numerous, and driven primarily by the limited availability of large numbers of patients to conduct definitive, pivotal clinical trials. Despite these complexities, several chronic CF therapies have been approved over the past two decades with the help of CF multicentre clinical trial networks, including the CF Therapeutics Development Network (TDN) and the European Clinical Trials Network (CTN). ${ }^{2}{ }^{3}$ Today, development efforts are expanding beyond chronic therapies targeting distal sequelae of pulmonary airway obstruction, infection and inflammation $^{4-7}$ to agents targeting proximal dysfunction associated with reduced epithelial anion transport resulting from mutant $\mathrm{CF}$ transmembrane conductance regulator (CFTR) protein. CFTR-enhancing therapies in development include inhaled agents intended to either express wild-type CFTR genes ${ }^{8}$ or to repair 'defective' CFTR RNA transcripts ${ }^{9}$ in the CF airway, and systemic single and combination agents (commonly referred to as 'modulators') intended to enhance mutant CFTR protein function. ${ }^{10}$

Most agents in development that are intended to increase CFTR function are tailored to treat specific CFTR mutations carried by individuals with CF, meaning that demonstration of their efficacy will require studies in specific patient subpopulations. Five broad CFTR functional mutation classes have been described: three classes (Classes I-III) are considered 'severe' in that they are associated with little or no protein function at the epithelial surface, and two are considered 'mild' functional classes (IV and V) where CFTR activity is reduced but not absent. Class I, II and III CFTR mutations are of the highest priority to modulator developers because they are associated with the greatest number of patients and worse clinical outcomes. ${ }^{11}$ Severe CFTR mutations comprise three dysfunctional aetiologies: Class I mutations are nonsense mutations resulting in incomplete mRNA transcripts and protein production, Class II are processing mutations where an altered protein product is incorrectly processed and fails to reach the cell surface, and Class III are gating mutations in which altered protein at the membrane surface has reduced ability to support anion transit. ${ }^{11}$ Correspondingly, different systemic CFTR modulator types address these aetiologies: 'read-through' agents allow ribosomes to continue translation through Class I nonsense mutations to produce full-length CFTR protein, 'correctors' facilitate Class II mutant CFTR protein processing and increase the quantity of CFTR protein at the plasma membrane and 'potentiators' increase the function of Class III mutant CFTR proteins residing at the plasma membrane. Importantly, some CFTR mutations display multiple dysfunctional aetiologies and so may require more than one type of agent for effective modulation.

While there has been encouraging progress in development of CFTR modulators, significant work remains. To date, no systemic modulators that have entered clinical trials have been genotype independent; all have been CFTR mutation dysfunction-specific. If CFTR modulators are to form the basis for personalised medicine in CF, effective modulators will be needed to treat the full range of CFTR genotypes found in the CF population. ${ }^{12}$ In addition, more potent alternatives to currently approved systemic CFTR modulators will be needed to increase the magnitude of benefit that can be achieved with respect to clinical outcomes. Finally, approval of more than one CFTR 
modulator for the same indication has the potential to introduce market competition that may motivate reductions in the substantial annual treatment costs associated with these therapies. ${ }^{13} 14$

Given the rapid development pace of CFTR modulator therapies when compared with other CFTR-enhancing strategies, we address in this paper the key issues that will impact our ability to advance new CFTR modulators through the drug development pipeline. The objectives of this review are to outline the challenges and opportunities in drug development created by genotype-specific CFTR modulators, highlight the advantages of sweat chloride as an established biomarker of CFTR activity to streamline early-phase development and summarise options for later phase clinical trial designs that respond to the adoption of approved genotype-specific modulators into standard of care. As the complexity of developing therapies in a competitive drug development environment and a finite patient population is not unique to CFTR modulators, many of the issues addressed in this paper can be extended to the entire spectrum of CFTR-enhancing therapies.

\section{THE CURRENT STATE OF CFTR MODULATOR AVAILABILITY}

To date, two small-molecule CFTR modulators have been evaluated in pivotal clinical trials in individuals with CF and specific mutant CFTR genotypes that have led to regulatory review and/ or approval. The potentiator ivacaftor has been approved in the US, Canada, Australia and the European Union for individuals $\geq 2$ years of age with the G551D CFTR gating mutation and, with the exception of Australia, other gating mutations including $\mathrm{R} 117 \mathrm{H}$. For perspective, these most prevalent gating mutations, G551D and R117H, represent approximately $7 \%$ of the US CF population. ${ }^{15}{ }^{16}$ Ivacaftor treatment of individuals with $\mathrm{CF}$ and at least one G551D allele is associated with a robust clinical response, including a $10.6 \%$ absolute improvement in $\mathrm{FEV}_{1}$ per cent predicted, a $55 \%$ reduction in pulmonary exacerbations over 24 weeks when compared with placebo ${ }^{16}$ and a reduced rate of $\mathrm{FEV}_{1}$ decline over 3 years when compared with matched homozygous F508del controls. ${ }^{17}$ The corrector-potentiator pair lumacaftor-ivacaftor has been approved in the US and is currently under review in the European Union for individuals $\geq 12$ years of age who are homozygous for the combined processing and gating CFTR mutation F508 del. ${ }^{18}$ This patient group represents approximately $34 \%$ of the US CF population, and a similar percentage in Europe. ${ }^{19}{ }^{20}$ Lumacaftor-ivacaftor treatment (ivacaftor with $400 \mathrm{mg}$ lumacaftor every $12 \mathrm{~h}$ ) is associated with a more modest $2.8 \%$ improvement in $\mathrm{FEV}_{1} \%$ predicted and a 39\% reduction in pulmonary exacerbations over 24 weeks when compared with placebo in F508del homozygotes. $^{21}$

Both ivacaftor and lumacaftor-ivacaftor are administered orally as opposed to by inhalation, with evidence of functional CFTR enhancement in tissues outside the airway, including the gastrointestinal tract and sweat gland. ${ }^{16} 2122$ Although systemic drug administration can have disadvantages associated with increased systemic exposure and potential for toxicity, important CF gastrointestinal complications have been attributed to CFTR dysfunction and there is good evidence that effective systemic CFTR modulation provides health benefits beyond the respiratory tract. ${ }^{22}$

\section{KEY CHALLENGES AND OPPORTUNITIES IN THE CLINICAL DEVELOPMENT OF NEW CFTR MODULATORS}

As the CF community maps out strategies for continued effective development of CFTR modulators, it is essential to consider emerging clinical development issues, some of which have not been encountered in CF before. The requirement of CFTR genotype-specific populations further narrows the already limited patient populations eligible for CF clinical trials beyond the normally imposed eligibility criteria such as lung disease severity and age, and makes simultaneous conduct of multiple modulator trials especially challenging. This unprecedented situation necessitates an even greater sensitivity towards study sample size requirements and feasibility, particularly for earlyphase studies that may have greater flexibility than later phase pivotal trials. The effective development of genotype-specific modulators therefore requires consideration of the relative availability of subjects for each CFTR genotype (figure 1), particularly if other therapies targeting the same genotype are concurrently in clinical trials. For modulators targeting genotypes with already approved modulators (dark bars of figure 1), trial designs are also complicated by long-term placebocontrolled comparisons being less likely to be ethical or feasible, particularly as available modulators become increasingly efficacious. Finally, as additional highly efficacious and genotypespecific modulators become adopted as standards of care, subsequent agents sharing the same or similar mechanisms and indications will likely need to be developed and evaluated as replacement therapies rather than as 'add-ons', unlike new mucolytic or anti-inflammatory agents in development. In some aspects, this situation is comparable with the current development landscape for new inhaled antibiotics in which there are effective inhaled antibiotics available to patients, chronic administration is a global standard of care for persons with CF and chronic Pseudomonas aeruginosa airway infection, ${ }^{23} 24$ and active comparative trials against inhaled tobramycin are now required for European Medical Agency (EMA) approval. ${ }^{25}$ As expanded upon in later sections, the use of an approved therapy as an active control may require increased sample sizes in comparison with a placebo control, and the acquisition of marketed agents may incur additional costs if blinding is required by regulators regardless of whether an existing therapy is considered standard of care.

As a balance to these challenges, however, attractive opportunities exist for any next-generation modulator that has the potential for substantially increased efficacy compared with approved therapies. One example of this is for newer modulator candidates targeting the F508del mutation. As noted previously, lumacaftor-ivacaftor is approved in the US for individuals with CF and two copies of F508del mutation, but not for individuals with only one F508del mutation. An important opportunity is that a modulator anticipated to have substantially greater clinical efficacy than lumacaftor-ivacaftor among F508del homozygotes might also be anticipated to have a modest but clinically significant effect on genotypes containing only one F508del mutation, a large unserved population (figure 1) in which placebo-controlled trials would be readily feasible today. Attention to variability in response in this more diverse population as a potential confounder in the assessment of efficacy will however be required. Once an efficacious modulator therapy is approved for individuals with a single F508del mutation, developers of subsequent CFTR-enhancing therapies (whether mutation-specific or not) would face similar challenges to those already outlined, albeit in a larger potential study population. Another opportunity is the very strong desire by the CF community to support development of additional modulators given the recognised variability in individual response and tolerance to currently approved modulators. ${ }^{26}$ 
Figure 1 Distributions of genotypes containing the 20 most prevalent mutant cystic fibrosis transmembrane conductance regulator (CFTR) alleles among individuals in the US with CF. Genotypes (each consisting of two CFTR alleles) are shown for individuals with CF followed in the 2012 US CF Foundation Patient Registry (CFFPR). ${ }^{19}$ The 20 most prevalent mutant CFTR alleles are shown in the same order on each axis with each possible genotype represented by only one bar and homozygous genotypes found on the front diagonal. The most prevalent genotype is F508del/F508del (far left). F508del compound heterozygotes are the remainder of bars along the first which there is currently an approved CFTR modulator are highlighted with dark bars. The same data are depicted in ( $A$ and $B)$, but with the $Y$-axis shown as linear in $(A)$ and $\log _{10}$ scale in (B). CFTR allele 'wall'. Those genotypes for
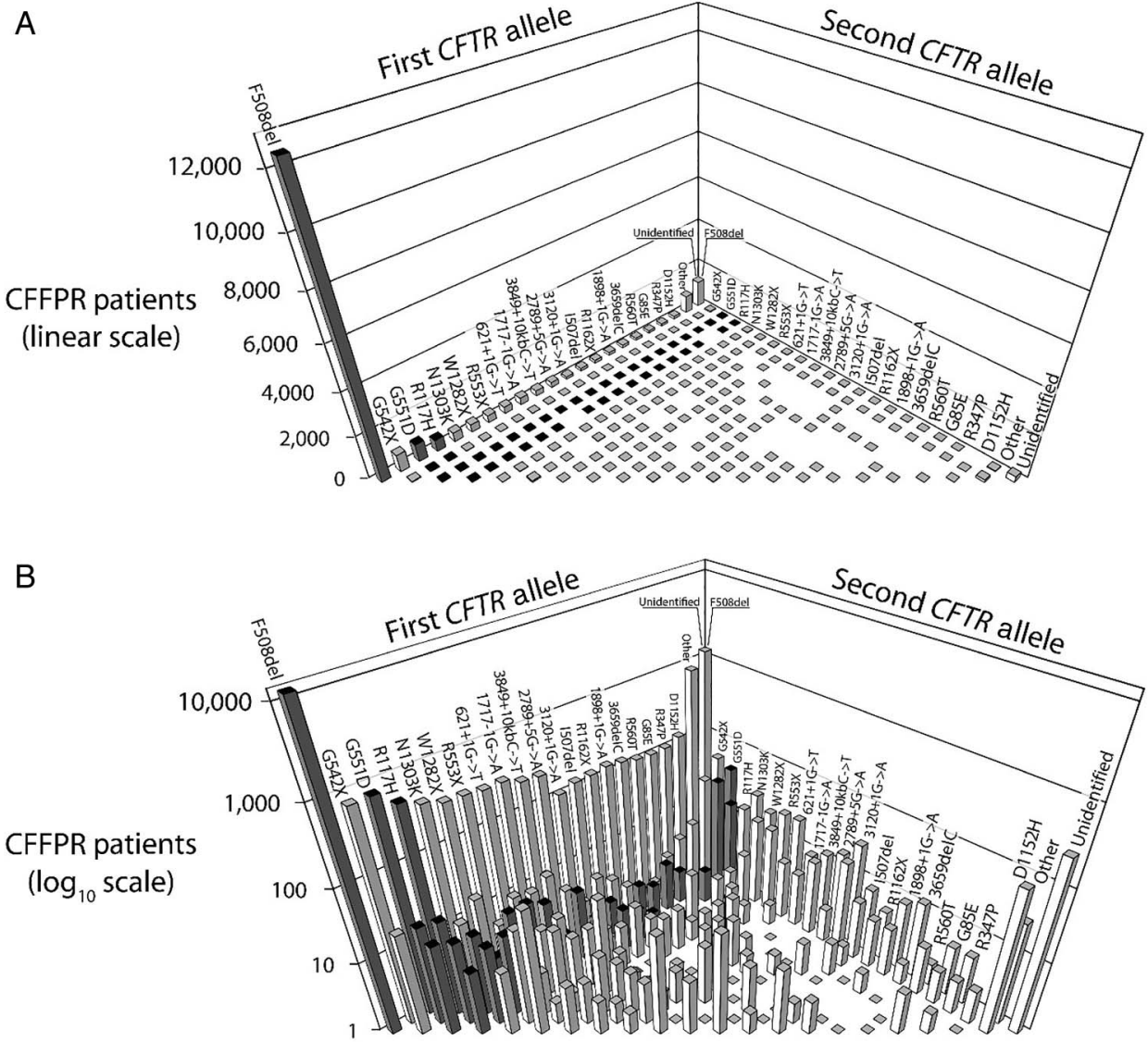

\section{ESTABLISHMENT OF CFTR-SPECIFIC ACTIVITY IN EARLY-PHASE STUDIES}

\section{Preclinical data to establish an effect on CFTR activity}

To assure optimal use of eligible patient populations, before proceeding to clinical trials modulator candidates should undergo a comprehensive preclinical assessment to confirm that proposed or early observed activity is associated with CFTR modulation that likely matches or exceeds currently approved therapies. Standardised in vitro assays afford comparison with data from other established modulators and allow assessment of the

Table 1 Cystic fibrosis biomarkers studied with approved cystic fibrosis transmembrane conductance regulator (CFTR) modulators

\begin{tabular}{llll}
\hline Biomarker & $\begin{array}{l}\text { Ivacaftor in } \\
\text { G551D }\end{array}$ & $\begin{array}{l}\text { Lumacaftor- } \\
\text { ivacaftor in F508del/ } \\
\text { F508del }\end{array}$ & References \\
\hline $\begin{array}{l}\text { Sweat chloride } \\
\begin{array}{l}\text { Nasal potential } \\
\text { difference }\end{array}\end{array}$ & $\sqrt{ }$ & $\sqrt{ }$ & $16,22,26,28-31$ \\
$\begin{array}{l}\text { Mucociliary } \\
\text { clearance }\end{array}$ & $\sqrt{ }$ & $22,26,28$ \\
$\begin{array}{l}\text { Duodenal pH } \\
\begin{array}{l}\text { Sputum } \\
\text { inflammatory }\end{array}\end{array}$ & $\sqrt{ }$ & 22 \\
$\begin{array}{l}\text { markers* } \\
\text { Lung clearance } \\
\text { index }\end{array}$ & $\sqrt{ }$ & 22 \\
$\begin{array}{l}\text { Exhaled N0 } \\
\text { Sweat rate }\end{array}$ & $\sqrt{ }$ & 22 \\
\hline *Free neutrophil elastase, interleukin 8, others. & \\
& $\sqrt{ }$ & 32 \\
\end{tabular}

likelihood and potential magnitude of benefit in subsequent clinical trials. Suggestions for preclinical modulator evaluation assay systems have been outlined. ${ }^{27}$ Importantly, modulator candidates with robust preclinical data that suggest at least comparable, and preferably superior, restoration of CFTR function when compared with currently approved modulators are the most likely to elicit the support of the research and patient community, and encourage broad participation and rapid recruitment in subsequent clinical studies.

\section{Streamlining early-phase modulator study designs using biomarkers to confirm CFTR activity}

To reduce the use of study participants and unnecessary exposure to candidate modulators entering first-in-human and CF trials, early-phase studies should have a primary focus on the evaluation of safety. Early-phase studies also serve an important role however in characterising the potential for clinical efficacy of a modulator candidate, including demonstration that all components of a combination are essential to overall efficacy and optimisation/rationalisation of modulator dosage and regimen. If conducted properly, these studies can dramatically reduce the risk of failure in subsequent phase III studies. However, these early-phase studies need not necessarily employ endpoints that actually demonstrate clinical efficacy, which in CF studies require relatively large sample sizes. Alternatively, limited resources can be spared by assessing treatment-associated changes in 'appropriate' biomarkers that require substantially fewer subjects but that are reasonable predictors of the potential for clinical efficacy in subsequent larger studies. Modulator candidates with strong preclinical data dossiers that include recommended in vitro CFTR assays ${ }^{27}$ will be most amenable to estimation of in vivo CFTR biomarker responses in phase II 
study populations. A number of CF biomarkers have been described with varying levels of sensitivity in measuring in vivo biological activity associated with approved CFTR modulators (table 1).

For systemic modulators, sweat chloride, nasal potential difference (NPD) and duodenal $\mathrm{pH}$ are the more direct measures of increased CFTR activity among the biomarkers listed in table $1 ; 2234$ others capture 'downstream' consequences of augmented CFTR function (as do clinical endpoints such as changes in $\mathrm{FEV}_{1}$ or pulmonary exacerbation risk). Among these three biomarkers, the value of duodenal $\mathrm{pH}$ and its relationship to subsequent clinical benefit are the least characterised. Although sweat chloride and NPD both demonstrated substantial and relatively rapid response to ivacaftor treatment, ${ }^{22} 2931$ collection of NPD data is considerably more complex than collection and analysis of sweat. In addition, only sweat chloride has demonstrated a significant yet attenuated response to lumacaftor-ivacaftor (figure 2), ${ }^{26}{ }^{28}$ suggesting that sweat chloride may be the most sensitive and best currently available direct biomarker measure for CFTR systemic modulators.

Change in sweat chloride has a number of attributes that fit well in phase II demonstration of efficacy potential (table 2). The measure is fully standardised, non-invasive, has good dynamic range and has relatively low associated variance and is sensitive to modulator effects within days of treatment initiation.

Another strength of sweat chloride listed in table 2 is its low variability relative to anticipated effect size, in contrast to biomarkers used to establish the mechanism of action for other CF therapeutic classes (eg, changes in bacterial density for inhaled antibiotics or neutrophil elastase for anti-inflammatories). ${ }^{35}$ This allows use of substantially lower sample sizes to demonstrate even modest effects. Table 3 highlights differences in sample sizes required in a 1:1 randomised, placebo-controlled prospective study to demonstrate a mean $10 \mathrm{mmol}$ reduction in sweat chloride versus a $3 \%$ predicted increase in $\mathrm{FEV}_{1}$ (both modest responses similar to those observed with lumacaftor-ivacaftor treatment in F508del homozygotes). ${ }^{21}{ }^{26}$ If a sweat chloride endpoint is used as a primary endpoint in comparison with a traditional $\mathrm{FEV}_{1}$ endpoint in early-phase development, required sample sizes can be substantially reduced. Maintaining $\mathrm{FEV}_{1}$ as a secondary endpoint in these studies will, in addition to monitoring safety, enable initial estimates of treatment effect and variability that can be used to design and power later phase studies. Phase II study designs using sweat chloride endpoints can also be considerably shorter than later phase clinical efficacy studies, improving the acceptability of placebo-controlled designs to the CF community and ethics review boards even in the setting of existing approved therapies.

The most notable shortcoming of sweat chloride as a CFTR biomarker may be its complicated relationship with long-term

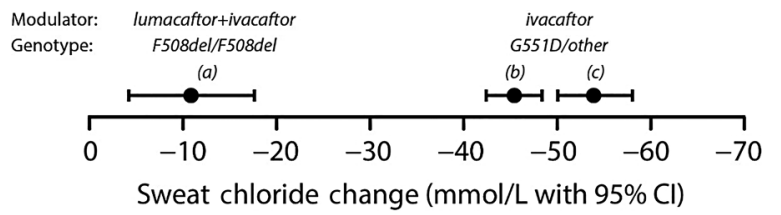

Figure 2 Mean treatment-associated changes in sweat chloride concentration observed in three trials of cystic fibrosis transmembrane conductance regulator (CFTR) modulators. Modulators and CF genotypes studied are identified above observed values. Studies $(A)^{26}$ and $(B)^{16}$ were blinded, randomised and placebo-controlled. Study $(C)^{22}$ was observational. Bars around point estimates represent 95\% Cls.
Table 2 Strengths and weaknesses of change in sweat chloride as a phase II biomarker for modulator candidates

\begin{tabular}{ll}
\hline Strengths & Weaknesses \\
\hline $\begin{array}{l}\text { Highly standardised test } \\
\text { Widely available }\end{array}$ & $\begin{array}{l}\text { Not a clinical endpoint } \\
\text { No } 1: 1 \text { correlation with a clinical } \\
\text { endpoint } \\
\text { Not measured at site of desired clinical } \\
\text { effect }\end{array}$ \\
$\begin{array}{l}\text { Non-invasive } \\
\text { Measure of effect on CFTR function }\end{array}$ \\
$\begin{array}{l}\text { Indicative of systemic drug } \\
\text { bioavailability } \\
\text { Rapid time to response (days) } \\
\text { Rapid washout (days) } \\
\text { Acceptable dynamic range (10- } \\
60 \text { mmol/L) } \\
\text { Low variability relative to effect size }\end{array}$ \\
\hline
\end{tabular}

CFTR, cystic fibrosis transmembrane conductance regulator.

clinical outcomes. Investigators have noted that sweat chloride change is not predictive of $\mathrm{FEV}_{1}$ change within individual patients, ${ }^{30}$ and two retrospective analyses of phase III ivacaftor study data have reached differing conclusions with respect to the strength of the association between these outcomes. ${ }^{36}{ }^{37}$ Discordance between the magnitude of modulator-associated sweat chloride change and $\mathrm{FEV}_{1}$ change in individuals may be due to differences in tissue bioavailability or efficacy thresholds in the sweat duct and the airway, and may also result from inhomogeneous 'reversibility' of CF lung dysfunction. In fact, variability of lung function response to other chronic respiratory therapies has tended to be the rule rather than the exception in randomised controlled trials. ${ }^{4-6}$ However, despite the lack of a direct linear correlation between $\mathrm{FEV}_{1}$ in individuals and sweat chloride, there is strong evidence that sweat chloride predicts clinical outcomes at the population level, ${ }^{38}$ supporting its role as a key outcome measure for clinical trials. Further evidence is needed to determine how helpful a sweat chloride endpoint will be in demonstrating modulator dose proportionality, but given that small changes in CFTR function are known to result in significant sweat chloride changes, there appears to be strong potential. ${ }^{29} 3940$ To be clear, because of the potential for tissuespecific effects, a systemic candidate modulator that does not decrease sweat chloride may still in theory have the potential to improve CF health, but when a candidate is shown to substantially decrease sweat chloride it is reasonable to predict that subsequent clinical benefit will be observed in larger phase III trials. In contrast, a candidate with a nominal sweat chloride effect may garner little enthusiasm for development from the community. Overall, the strengths of sweat chloride as a primary endpoint in early phase trials of systemic CFTR modulators appear to far outweigh its perceived weaknesses.

\section{Evaluating the contribution of components of modulator combinations}

It is likely that for some genotypes, a combination of two or three genotype-specific modulators will be required to reach high levels of CFTR restoration. ${ }^{34}$ Early-phase study designs will need to determine the efficacy contribution of each individual component of modulator combinations as well as accumulate sufficient safety data to permit combination-only phase III studies. However, there are additional patient-related factors that warrant consideration during study design. A key challenge 
Table 3 Comparison of sweat chloride and $\mathrm{FEV}_{1} \%$ predicted as phase II endpoints assuming thresholds of effect comparable with those observed for lumacaftor-ivacaftor

\begin{tabular}{lll}
\hline & $\begin{array}{l}\text { Change in sweat } \\
\text { chloride }\end{array}$ & $\begin{array}{l}\text { Change in } \mathrm{FEV}_{1} \% \\
\text { predicted }\end{array}$ \\
\hline $\begin{array}{l}\text { Hypothesised treatment effect (new } \\
\text { modulator-placebo) }\end{array}$ & $10 \mathrm{mmol} / \mathrm{L}$ & $3.0 \%$ predicted \\
Estimated SD* & 10 & 6.5 \\
Subjects per group $\dagger$ & 16 & 74 \\
Treatment duration & $3-7$ days & $14-28$ days \\
\hline *SD estimates of the 28 -day change in sweat chloride and $\mathrm{FEV}_{1} \%$ predicted derived \\
from prior trials. \\
†1:1 randomisation, two-sided t test with $80 \%$ power and two-sided 0.05 level of \\
significance.
\end{tabular}

of trials that evaluate only a single component of a modulator combination therapy will be patient hesitancy to participate in trials in which a test treatment is expected to be less efficacious than existing prescribed therapy. Much more attractive to patients will be trials designed to evaluate contribution of individual components of a combination of modulators while also allowing some participants access to a combination of modulators expected to be more efficacious than existing therapies.

In summary, early-phase modulator development should ideally (1) establish definitive CFTR modulation activity, (2) provide dose/regimen rationalisation for subsequent studies and evidence of the contribution of components of modulator combinations, (3) accumulate safety and tolerability data (4) and provide evidence of clinical efficacy potential, while keeping an emphasis on smaller study size and duration by using wellcharacterised biomarkers.

\section{DEMONSTRATING CLINICAL EFFICACY IN LATER PHASE STUDIES}

For late-phase clinical trials, developers must consider how to ethically conduct studies of new modulators when existing therapies may be available as standard of care. Figure 3 provides an overview of a study design framework that can be followed for later phase development of a new modulator monotherapy or combination therapy as dependent on target genotype indication, presence or absence of an approved modulator for the indication and anticipated efficacy. Unfortunately, there is no single 'ideal' recommendation on study design that can be made in this setting, as the optimal choice of study design is dependent, among other factors, on the flow of questions in figure 3.

By far the greatest study design divergence relates to an ability to conduct a placebo-controlled trial versus the necessity of conducting an active-comparator study. Even when an approved modulator exists for a particular CFTR genotype, there are circumstances (such as only modestly effective existing therapy) in which a placebo-controlled trial design could be used and would be the trial design of choice for establishing clinical efficacy due to its clear interpretability. ${ }^{41}$ Active-comparator studies should be proposed only after determining there is not an appropriate treatment-naïve population for which a placebocontrolled trial could be conducted (eg, international populations for which the drug is not approved or available), and when there is an unwillingness of patients or physicians to withdraw from current standard of care and be assigned to placebo for a given duration. An ongoing evaluation of the number of available and willing research participants will be critical to determine the necessity of active-comparator trials in CF as the landscape of new approved modulators changes. Below we address the key factors that will drive the feasibility of such active-comparator trials, while also motivating alternative study design approaches that could achieve greater feasibility and interpretability. We assume, based on precedence from other pivotal trials of CFTR modulators, ${ }^{16}{ }^{21}$ that change in $\mathrm{FEV}_{1} \%$ predicted will be the most widely accepted primary endpoint in this setting for which to establish efficacy.

\section{Active-comparator trials and alternative approaches}

When an active-comparator trial is necessary for candidate modulators intended to treat CFTR genotypes for which an effective genotype-specific agent is available, hypotheses and study designs are driven by whether the candidate is anticipated to have comparable or superior efficacy to the approved agent (figure 3). This choice will ideally be informed by available preclinical and phase II data. While candidate modulators with comparable efficacy will support market competition, those with superior efficacy (either independently, or with an additive or synergistic effect) will be most attractive to the CF community as they have the potential to further improve clinical outcomes. Although active-comparator trials enable evaluation of efficacy within the context of standard of care, there are three key potential challenges to this design in relation to a more traditional placebo-controlled trial: increased sample size requirements, potential costs incurred for acquisition and blinding of the active comparator (if blinding is required) and a less clear comparator group for interpreting safety data.

Using the precedent of change in $\mathrm{FEV}_{1} \%$ predicted as a primary endpoint for pivotal modulator trials, tables 4 and 5 provide an overview of the sample size requirements for various active controlled trial hypotheses. The magnitude of the sample size depends greatly on the anticipated effect size of the candidate versus the approved agent. As shown in table 4, when a new modulator is hypothesised to be superior to either an active comparator or placebo, the sample size requirements are quite reasonable. In some instances, such as a non-inferiority study against an approved agent with only modest efficacy, the design may be illogical from a clinical efficacy perspective (eg, risk of approving a drug, ie actually ineffective) or potentially infeasible (table 5, Scenario A). It is important to note that even in scenarios in which required sample sizes for the primary endpoint of $\mathrm{FEV}_{1}$ may be relatively modest, estimates are typically revised upwards during later phase development to improve power for secondary endpoints and increase likelihood of subsequent regulatory approval.

While there could conceivably be scenarios for which an active-comparator trial is feasible from a sample size perspective, an additional challenge to consider is active-comparator acquisition cost. Without methods for third party payers continuing to contribute to the costs of approved modulator therapies used in late stage trials, costs will be considerable for any blinded activecomparator study, and most expensive for active-comparator additive study designs for which the active comparator is needed in both study arms. Consider that a 'typical' safety and efficacy filing for a chronic CF therapy might include at least 200 subjects treated with a blinded active comparator for at least 6 months. Based on current acquisition costs for approved CFTR modulators, a potentially prohibitive sponsor investment would be required just to obtain the comparator for a pivotal active-comparator study. In an effort to enable blinded activecomparator studies and prevent financial barriers from impeding delivery of important therapeutic advancements, opportunities to shorten the required duration of pivotal trials and/or to 


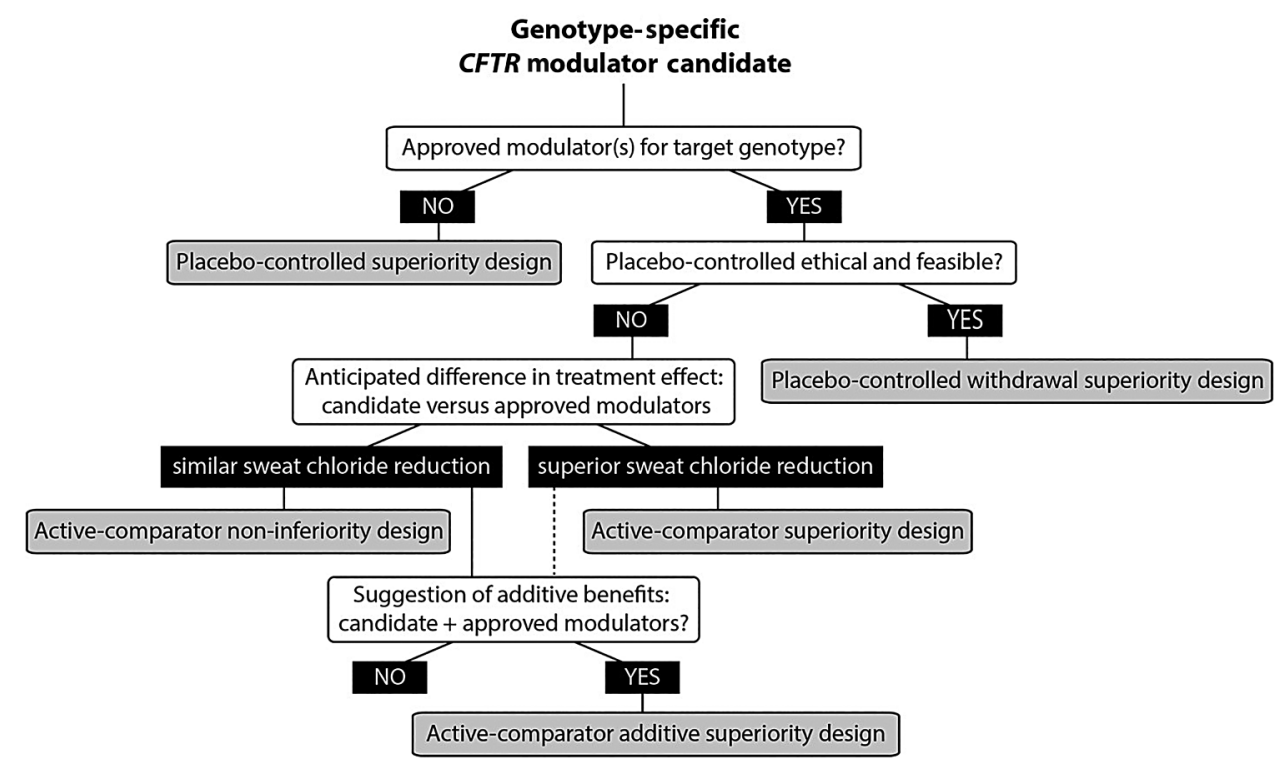

Figure 3 Possible pivotal trial study designs for a candidate cystic fibrosis transmembrane conductance regulator (CFTR) modulator as influenced by genotype target, the existing modulator landscape and anticipated clinical efficacy. For any candidate modulator and CFTR genotype pairing, a series of queries (white boxes) and responses (black boxes) will inform which of five study designs (grey boxes) might be considered.

collaborate with third parties to contribute to the costs of approved modulator therapies used in these trials need to be further explored. If open-label studies are acceptable to regulators and the active comparator is truly standard of care, designs in which one study group is randomly allocated to receive the new therapy and remaining subjects continue to receive standard of care, or designs in which subjects are randomly allocated to receive placebo or the new treatment in addition to the standard of care could dramatically reduce acquisition costs.

Given these challenges and the strong CF community interest in bringing additional modulators to market, there is clear motivation to identify 'creative' study design approaches to establish efficacy, which avoid the use of an active comparator. One alternative approach to streamlining studies of a new modulator with robust effect in a population receiving a comparable, approved modulator would be a 1-month 'withdrawal' design (figure 4). The hypothesis tested in such a design is

Table 4 Sample sizes for superiority study designs. The hypothesis is that the new modulator is more efficacious than a comparator (either placebo or an active comparator)

\begin{tabular}{|c|c|c|c|}
\hline & $\begin{array}{l}\text { Anticipated FEV } \\
\text { treatment effect: } \\
\text { New modulator } \\
\text {-comparator (\%) }\end{array}$ & $\begin{array}{l}\text { SD of the change } \\
\text { in FEV } \\
\% \text { predicted }^{*}\end{array}$ & $\begin{array}{l}\text { Sample size per } \\
\text { groupt }\end{array}$ \\
\hline $\begin{array}{l}\text { Scenario } \\
\text { A }\end{array}$ & 3 & 7.3 & 125 \\
\hline $\begin{array}{l}\text { Scenario } \\
\text { B }\end{array}$ & 5 & 7.3 & 45 \\
\hline $\begin{array}{l}\text { Scenario } \\
\text { C }\end{array}$ & 10 & 7.3 & 12 \\
\hline \multicolumn{4}{|c|}{$\begin{array}{l}\text { Scenarios estimate the sample size for a 1:1 randomised study with a primary } \\
\text { endpoint of absolute change in } \mathrm{FEV}_{1} \% \text { predicted. } \\
{ }^{*} \mathrm{SD} \text { approximated from the } 6 \text {-month changes in } \mathrm{FEV}_{1} \text { observed in previous } \\
\text { trials. }{ }^{1621} \\
\text { tEstimated using a two-sample } \mathrm{t} \text { test assuming } 90 \% \text { power and a two-sided } 0.05 \\
\text { level of significance. }\end{array}$} \\
\hline
\end{tabular}

whether the candidate modulator sustains $\mathrm{FEV}_{1}$ benefit over 4 weeks among subjects randomised to receive active treatment.

Although there may be ethical challenges to a withdrawal study in a population receiving a highly efficacious treatment, this may be mitigated through the use of a short duration study with predefined safety stopping rules. Further, sample sizes required to execute a study such as this with a superiority hypothesis are remarkably more manageable as compared with a non-inferiority study (table 4). A caveat to this proposed design is the need for robust data to estimate the effects and timing of wash-out of a chronic modulator therapy. Further, additional data on the clinical and biomarker effects of withdrawal from modulator therapy in CF are needed to help

Table 5 Sample sizes for active-comparator non-inferiority study designs. The hypothesis is that the new modulator is no more than a 'small amount' less efficacious than an active comparator, quantified by a non-inferiority (NI) margin

\begin{tabular}{|c|c|c|c|c|}
\hline $\begin{array}{l}\text { Previously } \\
\text { observed } \\
\text { FEV }_{1} \text { treatment } \\
\text { effect*: } \\
\text { comparator- } \\
\text { placebo, }(95 \% \mathrm{Cl})^{*}\end{array}$ & $\begin{array}{l}\mathrm{FEV}_{1} \mathrm{SD} \\
\text { of the } \\
\text { change in } \\
\mathrm{FEV}_{1} \\
\% \\
\text { predicted* }\end{array}$ & $\begin{array}{l}\% \text { of } \\
\text { Lowest } \\
\text { treatment } \\
\text { effect to } \\
\text { preserve }\end{array}$ & NI margint & $\begin{array}{l}\text { Sample } \\
\text { size per } \\
\text { group } ¥\end{array}$ \\
\hline
\end{tabular}

Scenario A: non-inferiority study vs an active comparator which has efficacy comparable with lumacaftor-ivacaftor

$3.0 \%(1.6$ to 4.4$) \quad 7.3 \quad 75 \% \quad 0.75 \times 1.6=1.2 \% \quad 778$

Scenario B: non-inferiority study vs an active comparator which has efficacy comparable with ivacaftor

$10.6 \%(8.6$ to 12.6$) \quad 7.0 \quad 50 \% \quad 0.50 \times 8.6=4.3 \% \quad 56$

* Treatment effects and SDs approximated from the 6-month changes in $\mathrm{FEV}_{1}$ observed in previous trials. ${ }^{1621}$

†The $\mathrm{NI}$ margin is derived based on preserving a percentage of the lowest possible treatment effect observed in the placebo-controlled trial, as captured by the lower bound of the $95 \% \mathrm{Cl}$. NI margins must be negotiated with regulators and ensure that a clinically meaningful effect size will be maintained.

¥Assuming there is truly no difference between the new modulator and the active comparator, sample size estimates are generated with $90 \%$ power to ensure that the lower limit of a one-sided $97.5 \% \mathrm{CI}$ will be above the NI margin. 


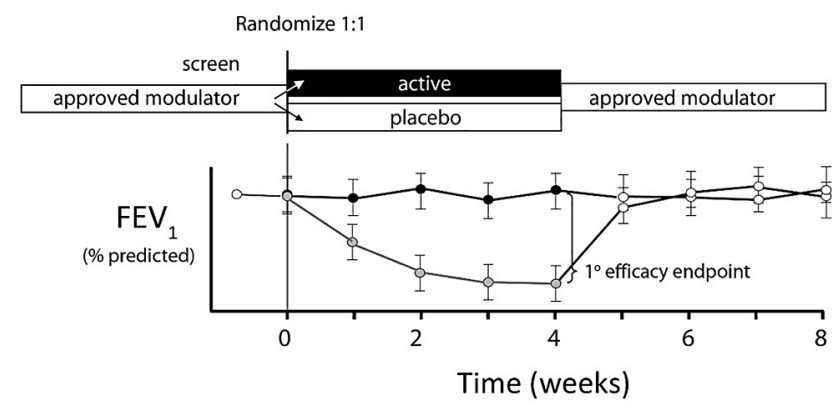

Figure 4 One-month washout design for a second-generation modulator studied in a population with access to a robust approved modulator. Subjects receiving the approved modulator are randomised $1: 1$ to receive the candidate modulator or placebo. Subjects can be optionally rolled over to active modulator for additional information.

determine the safety of such approaches, and clear rules for initiation of rescue therapy should be developed to limit the effects of exposure to either placebo or an ineffective therapy. Perhaps, the most compelling argument for designs that will enable a placebo comparison is that they avoid concerns over the unreliability of an active-comparator study to definitively establish effectiveness of a new therapy, in particular when the clinical efficacy of the approved therapy is modest or not clearly established itself. $^{41}$

Novel approaches to study design also include adaptive trial designs, which have not yet been fully used in the CF setting but enable more efficient dose ranging without significantly increasing sample size by having the trial design flexible and informed by accruing data, which enables early dropping of ineffective doses. ${ }^{42}$ These require complex designs, however, and further work is needed to determine the situations that

\section{Box 1 Outstanding modulator development issues} requiring regulatory input

Early-phase development issues

- Approaches to streamline phase II studies using biomarkers, particularly sweat chloride

- Methods of establishing the safety and role in efficacy for all components of modulator combinations

- Methods of efficiently demonstrating dose proportionality and selecting dose to be evaluated in later phase studies

Later phase development issues

- Study designs that enable feasibility of placebo-controlled study designs for genotypes with approved modulators

- Acceptability of abbreviated pivotal efficacy studies and washout study designs

- Acceptability of data from studies of subjects in countries where approved modulators are not available

- Active-comparator designs that minimise patient and financial resources

- Applicability of the combination rule for multicomponent modulators

Issues related to label extension

- Approval strategies in paediatric patients with healthy lung function that would benefit from prevention of lung disease

- Process for expanding modulator label indications to rare genotypes would most benefit from these approaches. Further efficiencies in study design can also be gained by the use of more formal interim monitoring of efficacy data throughout a trial using group sequential methods, which would enable early stopping (and hence reduction of sample size) for futility or efficacy/ harm. Group sequential monitoring has been used in CF trials previously and has led to the early stopping of trials in which early efficacy ${ }^{43}$ or futility ${ }^{44}$ have been observed.

\section{THE NEED TO ENGAGE WITH REGULATORS AND COLLECT ADDITIONAL DATA TO ENABLE EFFICIENT AND FEASIBLE DEVELOPMENT OF NEW CFTR MODULATORS}

Ultimately, clinical development success requires regulatory input at key milestones. Although some of the challenges for the development of CFTR modulators are not new to CF drug development, others including genotype-specific context, combination therapy guidelines and potential effect of cost on study design are unique. While the EMA has published guidelines for the clinical development of CF products, ${ }^{25}$ these predate the registration of CFTR modulators. In contrast to clear guidance for inhaled antibiotic study designs, current EMA guidelines provide efficacy endpoint recommendations but not suggested study designs for therapies intended to improve CFTR function. In box 1, we have outlined key development issues that will require regulatory input to provide guidance to the $\mathrm{CF}$ research community, as well as inform the additional evidence that may be needed to provide such guidance.

In addition to the issues outlined in box 1 , there is need for the CF community to in parallel obtain data that will enable us to objectively evaluate the feasibility of various study design approaches. These data will need to include robust estimates of available patient populations naïve to CFTR modulators as well as those already receiving these therapies, both within the US and internationally, to maximise the worldwide population of individuals with CF available for inclusion in studies. In addition, input from patients, families and CF healthcare providers on clinical equipoise for the use of placebo, and willingness to roll off of existing CFTR modulator therapies will be critical in the determination of need for placebo-controlled versus activecontrolled clinical trials. Data are also needed to determine expected clinical and biomarker response during short modulator wash-out periods, as these data will contribute to study designs involving placebo control in the presence of an already approved CFTR modulator.

Finally, expansion of approved modulator labels to include rarer CFTR genotypes (figure 1) is another important topic to be discussed with regulators. Due to the large ongoing effort to develop new systemic modulators for more prevalent CFTR genotypes, much of our focus has been on aspects of how studies in these populations can be reasonably executed. However, rare CFTR genotype populations are too small for traditional randomised studies, and thus alternative approaches must be discussed to enable individuals with rare mutations comparable access to safe and effective therapies.

\section{SUMMARY}

Although recent scientific breakthroughs offer the opportunity to dramatically alter the progression of CF through the use of novel CFTR modulator therapies, it is essential that proactive efforts be made now to identify drug development pathways that will assure continued advancement. We will need to develop efficient, feasible and regulatory-approved strategies to evaluate the safety and efficacy of the next generation of CFTR modulators while simultaneously allowing patients to maintain the benefits of 
modulators already incorporated into standard of care. Although strategies currently exist to streamline development, particularly in early phase studies, other strategies will require ongoing discussion and collaboration between developers, regulatory agencies and the $\mathrm{CF}$ community to assure the future delivery of disease-modifying drugs to those who most need them.

Acknowledgements The authors thank Eric Olson, Preston Campbell III, Chris Penland and Mary Dwight for critical review of the manuscript.

Contributors All authors contributed to drafting the manuscript for intellectual content.

Funding Supported by grants from the National Institute of Health grants P30DK089507 and UL1TR000423, and Cystic Fibrosis Foundation Therapeutics.

Competing interests NM-H reports grants from National Institutes of Health and the Cystic Fibrosis Foundation Therapeutics during the conduct of the study; she reports fees received by her institution outside the scope of this work from 12th Man Technologies, CURx Pharmaceuticals, Inc, Flatley Discovery Lab LLV, Insmed, Inc, KaloBios, Laurent Pharmaceuticals Corp, Mpex Pharmaceuticals, Inc, Nivalis Therapeutics, Inc, Nordmark, Novartis Pharmaceuticals Corp, Parion Sciences, Pharmaxis Ltd, Savara Pharmaceuticals and Vertex Pharmaceuticals, Inc. DVD reports personal fees from Vertex Pharma, AbbVie, Flatley Labs, Concert Pharma, Raptor Pharma, CURx Pharmaceuticals, Flatley Discovery Lab LLV, KaloBios, Genentech, Savara, Gilead Sciences, GlycoMimetics, Aradigm, Medimmune, Baxter Healthcare, Laurent Pharmaceuticals and the Cystic Fibrosis Foundation outside the submitted work. MB reports being a current employee of the Cystic Fibrosis Foundation and previous grants from Vertex Pharmaceuticals, outside the submitted work.

Provenance and peer review Not commissioned; externally peer reviewed.

Open Access This is an Open Access article distributed in accordance with the Creative Commons Attribution Non Commercial (CC BY-NC 4.0) license, which permits others to distribute, remix, adapt, build upon this work non-commercially, and license their derivative works on different terms, provided the original work is properly cited and the use is non-commercial. See: http://creativecommons.org/ licenses/by-nc/4.0/

\section{REFERENCES}

1 Armstead J, Morris J, Denning DW. Multi-country estimate of different manifestations of aspergillosis in cystic fibrosis. PLOS ONE 2014;9:e98502.

2 Rowe SM, Borowitz DS, Burns JL, et al. Progress in cystic fibrosis and the CF Therapeutics Development Network. Thorax 2012;67:882-90.

3 Döring G, Elborn JS, Johannesson M, et al. Clinical trials in cystic fibrosis. J Cyst Fibros 2007;6:85-99.

4 Fuchs HJ, Borowitz DS, Christiansen DH, et al. Effect of aerosolized recombinant human DNase on exacerbations of respiratory symptoms and on pulmonary function in patients with cystic fibrosis. The Pulmozyme Study Group. N Engl I Med 1994;331:637-42.

5 Ramsey BW, Pepe MS, Quan JM, et al. Intermittent administration of inhaled tobramycin in patients with cystic fibrosis. Cystic Fibrosis Inhaled Tobramycin Study Group. N Engl J Med 1999;340:23-30.

6 Retsch-Bogart GZ, Quittner AL, Gibson RL, et al. Efficacy and safety of inhaled aztreonam lysine for airway pseudomonas in cystic fibrosis. Chest 2009;135:1223-32.

7 McCoy KS, Quittner AL, Oermann CM, et al. Inhaled aztreonam lysine for chronic airway Pseudomonas aeruginosa in cystic fibrosis. Am I Respir Crit Care Med 2008;178:921-8. doi:10.1164/rccm.200712-18040C

8 Alton EW, Armstrong DK, Ashby D, et al. Repeated nebulisation of non-viral CFTR gene therapy in patients with cystic fibrosis: a randomised, double-blind, placebo-controlled, phase 2b trial. Lancet Respir Med 2015:3:684-91.

9 Brinks V, Lipinska K, Koppelaar M, et al. QR-010 treatment for cystic fibrosis: assessing the airway mucus barrier to delivery. Pediatr Pulmonol 2015;541:271.

10 Boyle MP, De Boeck K. A new era in the treatment of cystic fibrosis: correction of the underlying CFTR defect. Lancet Respir Med 2013;1:158-63.

11 Rowe SM, Miller S, Sorscher EJ. Cystic fibrosis. N Engl J Med 2005;352:1992-2001.

12 Bell SC, De Boeck K, Amaral MD. New pharmacological approaches for cystic fibrosis: promises, progress, pitfalls. Pharmacol Ther 2015;145:19-34.

13 O'Sullivan BP, Orenstein DM, Milla CE. Pricing for orphan drugs: will the market bear what society cannot? JAMA 2013;310:1343-4.

14 Orenstein DM, O'Sullivan BP, Quinton PM. Cystic fibrosis: breakthrough drugs at break-the-bank prices. Glob Adv Health Med 2015;4:8-57.

15 http://pi.vrtx.com/files/uspi_ivacaftor.pdf

16 Ramsey BW, Davies J, McElvaney NG, et al. A CFTR potentiator in patients with cystic fibrosis and the G551D mutation. N Engl J Med 2013;365:1663-72.
17 Sawicki GS, McKone EF, Pasta DJ, et al. Sustained benefit from ivacaftor demonstrated by combining clinical trial and cystic fibrosis patient registry data. Am J Respir Crit Care Med 2015;192:836-42.

18 http://pi.vrtx.com/files/uspi_lumacaftor_ivacaftor.pdf

19 Cystic Fibrosis Foundation Patient Registry. 2012 Annual data report to the Center Directors, Bethesda Maryland, 2013. Cystic Fibrosis Foundation.

20 Mehta G, Macek M Jr, Mehta A. European Registry Working Group. Cystic fibrosis across Europe: EuroCareCF analysis of demographic data from 35 countries. J Cyst Fibros 2010;9(Suppl 2):S5-21.

21 Wainwright CE, Elborn JS, Ramsey BW, et al. Lumacaftor-ivacaftor in patients with cystic fibrosis homozygous for Phe508del CFTR. N Engl J Med 2015;373:220-31.

22 Rowe SM, Heltshe SL, Gonska T, et al. Clinical mechanism of the cystic fibrosis transmembrane conductance regulator potentiator ivacaftor in G551D-mediated cystic fibrosis. Am J Respir Crit Care Med 2014;190:175-84. doi:10.1164/rccm. 201404-07030C

23 Mogayzel PJ Jr, Naureckas ET, Robinson KA, et al. Cystic fibrosis pulmonary guidelines: chronic medications for maintenance of lung health. Am J Respir Crit Care Med 2013;187:680-9.

24 Smyth AR, Bell SC, Bojcin S, et al. European cystic fibrosis society standards of care: best practice guidelines. J Cyst Fibr 2014;523-42.

$25 \mathrm{http} / /$ www.ema.europa.eu/docs/en_GB/document_library/Scientific_guideline/2009/ 12/WC500017055.pdf

26 Boyle MP, Bell SC, Konstan MW, et al. A CFTR corrector (lumacaftor) and a CFTR potentiator (ivacaftor) for treatment of patients with cystic fibrosis who have a phe508del CFTR mutation: a phase 2 randomised controlled trial. Lancet Respir Med 2014:2:527-38.

27 https://www.cff.org/Our-Research/Therapeutics-Development-Network/ Working-with-the-TDN/CFTR-Modulator-Compound-Review/

28 Clancy JP, Rowe SM, Accurso FJ, et al. Results of a Phase lla study of VX-809, an investigational CFTR corrector compound, in subjects with cystic fibrosis homozygous for the F508del-CFTR mutation. Thorax 2012;67:12-18.

29 Accurso FJ, Van Goor F, Zha J, et al. Sweat chloride as a biomarker of CFTR activity: proof of concept and ivacaftor clinical trial data. J Cyst Fibros 2014;13:139-47.

30 Barry PJ, Jones AM, Webb AK, et al. Sweat chloride is not a useful marker of clinical response to Ivacaftor. Thorax 2014;69:586-7.

31 Accurso FJ, Rowe SM, Clancy JP, et al. Effect of VX-770 in persons with cystic fibrosis and the G551D-CFTR mutation. N Engl J Med 2010;363:1991-2003.

32 Davies J, Sheridan $\mathrm{H}$, Bell $\mathrm{N}$, et al. Assessment of clinical response to ivacaftor with lung clearance index in cystic fibrosis patients with a G551D-CFTR mutation and preserved spirometry: a randomised controlled trial. Lancet Respir Med 2013;1:630-8

33 Grasemann H, Gonska T, Avolio J, et al. Effect of ivacaftor therapy on exhaled nitric oxide in patients with cystic fibrosis. J Cyst Fibros 2015;14:727-32.

34 Solomon GM, Marshall SG, Ramsey BW, et al. Breakthrough therapies: cystic fibrosis (CF) potentiators and correctors. Pediatr Pulmonol 2015;50(Suppl 40): S3-13.

35 Mayer-Hamblett N, Aitken ML, Accurso FJ, et al. Association between pulmonary function and sputum biomarkers in cystic fibrosis. Am J Respir Crit Care Med 2007; 175:822-8

36 Seliger VI, Rodman D, Van Goor F, et al. The predictive potential of the sweat chloride test in cystic fibrosis patients with the G551D mutation. J Cyst Fibros 2013:12:706-13.

37 Durmowicz AG, Witzmann KA, Rosebraugh CJ, et al. Change in sweat chloride as a clinical end point in cystic fibrosis clinical trials: the ivacaftor experience. Chest 2013;143:14-18

38 Heltshe SL, Mayer-Hamblett N, Rowe SM. Understanding the relationship between sweat chloride and lung function in cystic fibrosis. Chest 2013;144:1418.

39 Sosnay PR, Siklosi KR, Van Goor F, et al. Defining the disease liability of variants in the cystic fibrosis transmembrane conductance regulator gene. Nat Genet 2013:45:1160-7.

40 Masica DL, Sosnay PR, Raraigh KS, et al. Missense variants in CFTR nucleotide-binding domains predict quantitative phenotypes associated with cystic fibrosis disease severity. Hum Mol Genet 2015;24:1908-17.

41 Temple R, Ellenberg SS. Placebo-controlled trials and active-control trials in the evaluation of new treatments. Part 1: ethical and scientific issues. Ann Intern Med 2000;133:455-63.

42 Meurer WJ, Lewis RJ, Berry DA. Adaptive clinical trials: a partial remedy for the therapeutic misconception? JAMA 2012;307:2377-8.

43 Gibson RL, Emerson J, McNamara S, et al. Significant microbiological effect of inhaled tobramycin in young children with cystic fibrosis. Am J Respir Crit Care Med 2003;167:841-9.

44 Lechtzin N, West N, Allgood S, et al. Rationale and design of a randomized trial of home electronic symptom and lung function monitoring to detect cystic fibrosis pulmonary exacerbations: The early intervention in cystic fibrosis exacerbation (elCE) trial. Contemp Clin Trials 2013;36:460-9. 